\title{
Percepción del alumnado de grado y postgrado en la tutorización de trabajos fin de grado y fin de máster
}

\author{
Isabel M. Gomez-Trigueros \\ Facultad de Educación, Dpto. de Didáctica General y Didácticas Específicas, Universidad de Alicante, Aeroplano s/n, \\ San Vicente del Raspeig. Alicante-España. (correo-e: isabel.gomez@ua.es)
}

Recibido Jun. 23, 2020; Aceptado Ago. 25, 2020; Versión final Sep. 15, 2020, Publicado Feb. 2021

\begin{abstract}
Resumen
El objetivo de este estudio es analizar las percepciones acerca de la tutorización recibida por los estudiantes del Grado en Maestro de Primaria y del Máster del Profesorado de Secundaria de la Facultad de Educación de la Universidad de Alicante (España). La investigación se focaliza en el estudio de la dimensión personal y académica de sus tutores. Se ha seguido una metodología mixta y cuantitativa, aplicando un cuestionario ad hoc a 162 estudiantes y a través de entrevistas personales. Los resultados manifiestan la relevancia de la tutorización para dar respuesta a necesidades académicas, docentes, personales y profesionales. Se comprueba la necesidad de formar la dimensión personal de los tutores. Asimismo, se observa la necesidad de formar en competencias digitales docentes para mejorar la comunicación estudiante-tutor. Se concluye que los estudiantes participantes se sienten contentos con la función tutorial recibida a lo largo de la elaboración de su trabajo fin de grado y/o su trabajo fin de máster.
\end{abstract}

Palabras clave: educación superior; tutorización; universidad; formación de profesorado

\section{Undergraduate and graduate student perception of tutoring in final bachelor degree and final master's projects}

\begin{abstract}
The primary objective of this study is to assess tutoring perceptions by students in the primary education degree and in the master's degree in high school teaching from the faculty of education at the University of Alicante (Spain). The present research focuses on examining personal and academic dimensions of student tutors. A mixed quantitative methodology is applied by using an ad hoc questionnaire to 162 students and personal interviews. The results show the relevance of tutoring to successfully meet the demands of academic, teaching, personal, and professional needs. The results also show the need to train the personal dimension of tutors. There is also a need to train in digital teaching skills to improve student-tutor communication. It is concluded that the students surveyed are satisfied with the tutoring received during their final degree project and/or final master's project.
\end{abstract}




\section{INTRODUCCIÓN}

La adecuación de las universidades españolas al Espacio Europeo de Educación Superior (EEES) abarca diversos ámbitos tales como: la estructuración de titulaciones, la revisión de metodologías docentes, la actualización de contenidos, los nuevos enfoques de enseñanza-aprendizaje centrados en el estudiante (Ortega-Sánchez y Gómez-Trigueros, 2019), la delimitación curricular propuesta por competencias, la correcta integración de las tecnologías a través de la confección de entornos personales de aprendizaje entre otros. En tal contexto, también la función tutorial se ha visto transformada y enriquecida ante estos los nuevos requerimientos y directrices. Si bien es cierto que, históricamente, la orientación tutorial ha transitado desde la confección de diagnósticos situados en acciones puntuales, de tipo didáctico, humanista o laboral; en la actualidad, y de una manera progresiva, la percepción de la tutorización de calidad se ha ampliado, buscando dar respuesta a nuevas necesidad del alumnado (Winder et al., 2016; Amor-Almedina y Dios-Sánchez, 2017; Lee y Choi, 2017).

El tratado de Bolonia, demanda un nuevo rol para el docente universitario al que, además de sus obligaciones como enseñante, se incorpora la función de guía personal del estudiante. El profesorado-tutor se ve abocado a una formación y una cualificación tanto personal como académica, que dé respuestas y oriente al alumnado en su formación académica y profesional (López-Gómez, 2017; Alonso-García et al., 2018). De este modo, surgen estudios relacionados con la detección de aquellos factores y elementos que caracterizan al tutor/a y al proceso de tutorización de calidad. Algunos de estos trabajos han considerado que la tutorización de calidad es un elemento más del proceso educativo (Chianese, 2017), focalizándose en la formación instruccional y laboral del alumnado.

Otros estudios han centrado su análisis en la percepción de los dos elementos del binomio tutor/a-estudiante, centrándose en las dimensiones afectiva o interpersonal (Phillips y Wolcott, 2014; Dunworth y Sánchez, 2016; Lee y Choi, 2017) y valorando positivamente el efecto de modificación y de transformación del alumnado, receptor de la acción del tutor/a. En esta misma línea, la calidad del rol tutor/a debe poder medirse. Para ello es necesario constatar los cambios innovadores en tres ámbitos del asesoramiento implementado por el docente: el académico, el personal y el profesional. Por otra parte, se ha considerado que los factores y elementos que definen la correcta tutorización irían orientados a la generación de modelos adaptados a los distintos contextos educativos, que produzcan una mejora generalizada del tutorando (De Pablos et al., 2007). A tales consideraciones, Gaughf y Foster (2016) añaden el avance progresivo del profesorado-tutor/a hacia una orientación preventiva, sistemática, general y continuada destinada a promover el desarrollo integral del estudiante.

En todos los casos citados se pone en valor el importante papel de la innovación y la modernización como características que debe tener un profesional, que ofrezca una orientación y tutorización de calidad (Gros y Lara, 2009; Gómez-Trigueros et al., 2019). Al mismo tiempo, al tratarse de un proceso enmarcado en unas coordenadas espacio-temporal como es, para el caso de nuestra investigación, la elaboración de su Trabajo Fin de Grado (TFG) y/o su Trabajo Fin de Máster (TFM), la acción tutorial debe asentarse en uno principios concretos, claves, como son: a) de desarrollo personal: incidiendo en aspectos relativos a las competencias adquiridas y necesarias para dar respuesta a las cuestiones relacionadas con su TFG o TFM; sirviendo dicha orientación para facilitar el desarrollo individual, su propio auto concepto, la clarificación de valores y el desarrollo para la toma de decisiones sobre su investigación y cómo abordarla; b) de intervención educativa: orientada al diseño de su estudio y a cómo afronta el alumnado su realización; a la toma en consideración de sus circunstancias particulares, sus posibilidades técnicas y a su propia formación como graduados; c) de capacitación laboral: apoyando la propia dinámica que seguirán, en un futuro, como docente y posible tutor/a; proporcionando recursos (emocionales, formativos y bibliográficos) para solventar situaciones concretas en su labor profesional; etc. (ChanLin, 2016; Alonso-García et al., 2018, 64).

El Informe Universidad 2000 insiste en algunos de estos aspectos, definiendo la formación del tutor/a universitaria como: un asesor del estudiante antes y después de su ingreso en el centro; preparado y capaz de poner en práctica habilidades educativas; un planificador de programas y currículos didácticos; un apoyo en el caso de conflictos en el aula o de dificultades particulares del alumnado; un orientador en el desarrollo y en la madurez formativa y profesional de los estudiantes entre otros aspectos (Mouraz y Sousa, 2016; Lee y Choi, 2017; Tralongo, 2017). También, las directrices europeas indican tres niveles de atención para la tutorización en el ámbito universitario (Arco et al., 2009): 1) el personal; 2) el académico y 3) el profesional del estudiante. En este sentido, la tutorización de calidad debería abarcar la formación del tutor/a en cuestiones: burocráticas y logístico-administrativas; de orientación y motivación personal; profesionales y académicodocentes.

Aunque parece claro que todas estas características son identificativas de una función tutorial de calidad, no resulta sencillo identificar a profesionales que las ejemplifiquen. Entre los problemas para su detección se encuentran diversas razones. De un lado, el escaso conocimiento del profesorado universitario y más 
específicamente de los tutores sobre cómo ejercer su labor de orientación pues, el elevado número de departamentos así como el elevado volumen de docentes asociados, hace difícil que exista una identificación de aquellos profesionales que cuenten con una correcta predisposición para la función tutorial. Asimismo, la formación de los profesores tutores es otra de las dificultades a la hora de contar con profesionales de la función tutorial. A pesar de que las universidades recogen en sus planes de trabajo, y en su gestión de coordinación del profesorado, la necesidad de formar a los docentes en temas relacionados con la acción tutorial, la gran mayoría no ha recibido formación sobre cómo actuar para llevar a cabo una correcta acción tutorial de calidad (Ortega-Sánchez y Gómez-Trigueros, 2019). En este sentido, se adolece de estudios que investiguen la mala praxis tutorial en relación al alumnado universitario, cuestión que se presenta como esencial para lograr la mejora de la orientación universitaria (Dopico, 2013).

A todo esto se une la ausencia del reconocimiento (institucional y económico) de la labor tutorial, que da lugar a una falta de compromiso y una clara pasividad del profesorado en relación a su labor docente-tutor/a (Hounsell et al., 2008). En este entorno, que caracteriza a la universidad, se debe añadir la idiosincrasia de la tutorización de TFG y TFM, que suponen la finalización de una etapa de estudios (Grado y Máster respectivamente). En este sentido, la tutorización orientada a la dirección de un TFG o TFM debe conducir al estudiante en el proceso de búsqueda, análisis, gestión y evaluación de la información (De la Cruz et al., 2006). Además, debe ayudar a emprender la investigación, implementar métodos adecuados por medio de herramientas y estrategias, que proporcionen la resolución a las preguntas de la investigación; debe guiar al alumnado para que analice e interprete los resultados contrastándolos con la teoría, propiciando que identifique los alcances y limitaciones del estudio así como la confección de nuevas hipótesis, de otros problemas de su investigación y sus posibles aplicaciones (Hernández et al., 2016, 51). Pero todo esto no es suficiente, los estudiantes de TFG y TFM demandan una tutorización en la que se contemple, como elemento de calidad, el apoyo mutuo y las relaciones saludables en el binomio tutor/a-tutorando/a (Lechuga, 2011); orientado a transformar a los estudiantes poco experimentados en personas calificadas, para resolver problemas mediante su incorporación a redes de investigación en el campo de estudio (De la Cruz et al., 2006); basado en un intercambio profesional entre individuos con diferentes niveles de experiencia y conocimiento con el propósito de promover su desarrollo profesional y personal (Díaz y Bastias, 2012; Hernández et al., 2016; Gómez-Trigueros et al., 2019).

En este sentido, esta investigación se ha basado en el análisis de las necesidades del alumnado y profesorado en lo referente a la orientación y función tutorial de Grado y Postgrado de la Facultad de Educación; analizando la percepción de los factores y elementos que determinan la calidad de la tutorización según el alumnado, así como la detección de buenas prácticas por parte de los tutores de TFG y TFM participantes en el estudio. En base a esta contextualización y atendiendo al objetivo principal de la acción tutorial que es "favorecer la excelencia académica, la formación integral y la atención de todos los estudiantes" (Pantoja y Campoy, 2009, 18), este estudio analiza qué factores y elementos determinan una orientación y tutorización de calidad en los TFG y TFM, percibidos por el alumnado participante como principal receptor este servicio universitario.

\section{METODOLOGÍA}

El trabajo metodológico implementado en este trabajo ha sido de carácter mixto, a partir del uso de diferentes herramientas explicitadas en sucesivos epígrafes y suministrado a una muestra significativa de estudiantes.

\section{Participantes y contexto}

El contexto de la investigación ha sido la Facultad de Educación de la Universidad de Alicante (España) a lo largo de cuatro cursos académicos que extienden desde 2014-2015 hasta 2018-2019. Accedieron a participar en la investigación un total de 162 estudiantes de las asignaturas de "Trabajo Fin de Grado" de $4^{\circ}$ curso del Grado en Maestro en Educación Primaria y de Maestro en Educación Infantil así como de la asignatura de "Trabajo Fin de Máster" de la especialidad 9, Geografía e Historia del Máster del Profesorado de Secundaria. Se trata de grupos naturales (Hernández et al., 2010) conformados a partir de la organización de la propia Facultad de Educación. Su distribución por sexo fue de 93 mujeres (57.1\%) y de 69 hombres (42.9\%). La edad se encuentra comprendida entre los 21 y 24 o más 45 años, distribuidos en cuatro subgrupos (Tabla 1).

En relación a la asignatura cursada, los participantes se distribuyen en 22 estudiantes de la asignatura de TFG en Educación Infantil (13,5\%); 75 participantes (46,4\%) de la asignatura de TFG en Educación Primaria y 65 estudiantes de la asignatura de TFM (40,1\%), del Máster del Profesorado de Secundaria. La muestra es significativa respecto de los estudiantes que forman parte de la población, obteniéndose un índice de respuesta del $35,12 \%$ superando, por tanto, el $30 \%$ de respuesta necesario para la representatividad de estudios por encuesta (Hernández et al., 2010). 
Tabla 1. Datos de la muestra por sexo, edad y asignatura.

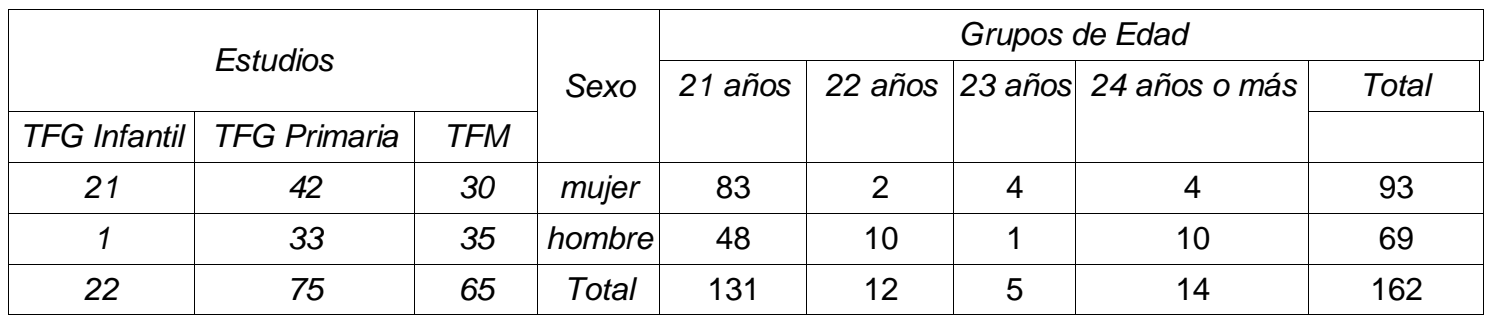

\section{Instrumento}

Se ha optado por un diseño metodológico mixto, basado en los estudios tipo cuestionario (o instrumento 1) y una entrevista personal (o instrumento 2) con la finalidad de analizar las percepciones de los estudiantes del TFG y TFM en relación a qué características son las ideales para valorar la acción tutorial de calidad, que permita el desarrollo personal, académico y profesional del alumnado. El cuestionario diseñados ad hoc, a partir de los empleados por Alonso-García et al. (2018) y Ortega-Sánchez y Gómez-Trigueros (2019), ha sido validado por expertos de diversas universidades públicas españolas e internacionales para el presente estudio, y se ha alojado en la aplicación de GoogleForms (https://forms.gle/pYFhK3kXfnLtaAD87). El instrumento consta de 29 ítems, en donde se combina la escala Likert de cinco puntos (1, Muy en desacuerdo - 5, Totalmente de acuerdo) y la respuesta abierta (sólo ítem 29) así como los relativos a cuestiones sociodemográficas (sexo, edad, estudios que ha realizado y TFG o TFM desarrollado ítems del 1 al 4). El tiempo de respuesta por parte de los participantes en la investigación ha sido de diez días y la recolección de datos se hizo en el mes de junio en todas las convocatorias por tratarse del periodo en el que se finaliza la función tutorial. Para dar respuesta a los objetivos de la investigación, se han realizado tanto análisis descriptivos como inferenciales, utilizando como herramienta el programa estadístico SPSS v.24.

Por su parte, se han llevado a cabo entrevistas personales semiestructuras. La selección y aplicación de la técnica de la entrevista tuvo como objetivo la obtención de información personalizada sobre las actitudes y representaciones de la práctica de acción tutorial para los participantes. La aplicación de esta técnica fue complementada con la realización del cuestionario arriba significado, con el fin de construir significados y profundizar en la interpretación de las percepciones individuales de los estudiantes (Hernández et al., 2010; Zohrabi, 2013). Partieron de un diseño semiestructurado de un total de diez preguntas-eje que permitieron acotar la información que se esperaba obtener y devolver información rica en matices. Los entrevistados y entrevistadas fueron convocados mediante correo electrónico institucional; se realizaron en la Facultad de Educación de la Universidad de Alicante a lo largo de la tercer y cuarta semana de junio de todos los cursos escolares del estudio. Tanto en los correos como en el inicio de la entrevista se informó a los participantes sobre las condiciones en que se desarrollaría, los objetivos de investigación y su duración: 55 minutos. Después de la explicación del propósito de investigación, se recordó a los participantes la confidencialidad y anonimato con los que se procesarían e interpretarían los datos obtenidos y que, además, serían grabados en audio.

Respecto al segundo instrumento de estudio, la entrevista individual, la información obtenida se sometió a una lectura exploratoria-comparativa detenida del contenido disponible, con el objeto de reducir las unidades de análisis a unidades de registro significativas e identificar las líneas temáticas y las posibilidades de saturación de las potenciales categorías y subcategorías-variables emergentes (Corbin y Strauss, 2015). Seguidamente, a partir de la comparación constante de los datos, se llevó a cabo un proceso de codificación abierta de las transcripciones. A través de un proceso de codificación axial, se agruparon y organizaron los códigos obtenidos, a partir de sus conexiones, en variables de estudio o subcategorías y se procedió a su codificación selectiva, por categorías, integradas en torno a cinco dimensiones de análisis centrales: dimensión personal; dimensión académica; dimensión burocrático-administrativa; dimensión comunicativa; y dimensión técnica. Finalmente, identificada la existencia parcial de más de una variable de estudio en las declaraciones expresadas por los estudiantes, las variables de análisis fueron codificadas y cuantificadas mediante una escala ordinal de 1 a 3 , donde 1 representa un bajo nivel de aproximación y 3 un alto nivel de aproximación a la variable objeto de estudio. Este procedimiento permitió ajustar, cuantitativamente, las tendencias explicativas de cada variable emergente (Tabla 3).

\section{Procedimiento de evaluación del instrumento y análisis de datos}

Con el fin de verificar la fiabilidad del cuestionario, se ha calculado el coeficiente alpha de Cronbach. El resultado obtenido $(\alpha=.891)$ constata la existencia de una adecuada consistencia interna del instrumento para el estudio propuesto (Hernández et al., 2010). Para dar respuesta a los objetivos de la investigación, se han realizado análisis descriptivos ( $\mathrm{M}=$ media; $\mathrm{DT}=$ desviación) e inferenciales. Se comprobó la no normalidad de los valores después de aplicar la prueba Kolmogorov-Smirnov, donde se constata que el nivel de significación es menor a 
0.05 ( $p<0.05)$, confirmando la distribución atípica de los datos. Tras el análisis descriptivo de los ítems, se hallaron correlaciones entre los aspectos analizados a través de la prueba Rho de Spearman $(p)$, estadístico no paramétrico, para medir la fuerza y la dirección de la asociación entre las variables clasificadas. Aparte de permitir conocer el grado de asociación entre variables, con Rho de Spearman es posible determinar la dependencia 0 independencia de dos variables aleatorias (Wilcox, 2011).

Con el objeto de comprobar los niveles de pertinencia y adecuación, grado de coherencia interna e importancia de la construcción de las entrevistas personales propuestas en el contexto de los objetivos de la investigación, se empleó el método Delphi (Landeta, 1999). De acuerdo con este método, fueron seleccionados expertos en diversas áreas (Sociología, Pedagogía y Psicopedagogía) para que pudieran emitir sus juicios, en una escala de intervalo de 5 puntos, sobre la pertinencia y adecuación, la coherencia interna y la importancia de las cuestiones que pretendían formularse, y su valoración general del constructo en una escala de 1 a 10 puntos.

La estabilidad de los resultados fue alcanzada, tras su consenso, después de administrar el instrumento de evaluación en dos rondas y obtener resultados estadísticamente fiables (criterio de parada). Se conformaron dos grupos: un grupo coordinador, constituido por los investigadores del presente estudio y responsables del diseño del instrumento, y el grupo experto evaluador, formado por docentes de las áreas arriba mencionadas. En relación a la estructura de la entrevista personal, los resultados finales obtenidos arrojan una media superior a 8 en la valoración general del constructo en cuanto a pertinencia y adecuación, coherencia interna e importancia de las cuestiones diseñadas así como una baja dispersión de las respuestas (DT $\leq 0.251$ ). También, se llevó a cabo un análisis de fiabilidad y consistencia interna del total de la escala $(\alpha=.902)$. Igualmente, los valores obtenidos en cada elemento de la escala se encuentran comprendidos, satisfactoriamente, entre $\alpha=.812$ y $\alpha$ $=.902$.

Identificados los patrones de contenido y categorizada la información disponible, se contabilizaron las palabras clave y la frecuencia de las respuestas temáticas más recurrentes, y se registraron los porcentajes de los fragmentos textuales concomitantes. Para la presentación de resultados, se vinculan los conceptos de análisis con los textos-respuesta más representativos de cada categoría. Para la identificación de los participantes, la organización de las unidades de registro y la presentación de los resultados, se ha procedido a la codificación alfabética de la actividad realizada y a la identificación alfanumérica de los estudiantes. Para el análisis de los datos cualitativos, se utilizó el software MAXQDA (v. 2020), por su capacidad para la codificación, categorización, saturación informativa, contabilización de palabras clave e interpretación de la información obtenida.

\section{RESULTADOS Y DISCUSIÓN}

Se presenta una selección de los resultados obtenidos en este trabajo para conocer las percepciones del estudiante universitario que elabora su TFG o su TFM acerca de la tutorización recibida. Para ello, el cuestionario confeccionado para esta investigación analiza aspectos o dimensiones referentes a la atención personal recibida, a la información proporcionada, a la orientación académica, a la resolución de cuestiones burocráticas y al asesoramiento profesional proporcionados durante la acción tutorial. Asimismo, valora la percepción de los participantes sobre la utilidad de las respuestas ofrecidas así como la cualificación de sus tutores/as para dar respuesta a las necesidades concretas en la elaboración de su TFG o TFM.

Si se observan los valores descriptivos (M y DT) (Tabla 2) de las respuestas del alumnado se constatan diferencias entre las dimensiones analizadas. Así, en los ítems que hacen referencia a la comunicación y rapidez en las respuestas por parte del tutor/a (ítems 5, 6, 18, 21 y 25), la opción de respuesta mayoritaria ha sido el valor 3 de la escala Likert que coincide con la respuesta "Ni de acuerdo ni en desacuerdo" (M $\leq 3.24$; DT 5 0.69). De igual forma, los ítems relativos a las cuestiones de índole burocrático o administrativo (ítems 8 y 17) arrojan un valor de respuesta "En desacuerdo" ( $\mathrm{M} \leq 2.12$; $\mathrm{DT} \leq 0.61)$, indicativo de la escasa formación del profesorado tutor/a en esta dimensión. Respecto a las preguntas sobre aspectos personales tales como empatía (ítem 12), conexión emocional (ítem 13), las respuestas se aproximan al valor "Ni de acuerdo ni en desacuerdo" ( $\mathrm{M} \leq 2.88$; $\mathrm{DT} \leq 0.81)$, poniendo de relieve un vacío existente respecto a las necesidades que presenta los estudiantes en cuanto a necesidades psicológicas y emocionales en el desarrollo de sus TFG o TFM. Tales resultados contrastan con las respuestas dadas a los ítems sobre la percepción personal del tutor/a en cuanto a confianza y seguridad transmitida (ítems 7, 14 y 15), motivación proporcionada (ítem 19), con una respuesta "De acuerdo" (M $4.45 ; \mathrm{DT} \leq 0.68)$.

En relación al seguimiento de los tutorandos/as, de la orientación y progreso desarrollado en la confección de sus trabajos (ítems 10,11, 22, 23, 26), las respuestas emitidas por los participantes han sido positivas, con un mayor número de respuestas "De acuerdo" (M 4.45 ; DT $\leq 0.67$ ), íntimamente relacionada con la adecuada gestión de los tutores/as en el control y evaluación del cronograma marcado por la institución universitaria. También, las respuestas han sido muy positiva sobre la acción tutorial en conocimientos académicos (ítem 16) y respecto de la valoración general del tutor/a asignado (ítems 9 y 27), que es capaz de reconocer sus 
propias limitaciones a la hora de ayudar al tutorando (ítem 20), al tiempo que promueve las fortalezas de los

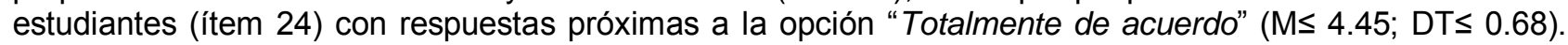
Destaca la opción de respuesta positiva sobre la acción tutorial en la orientación profesional (ítem 28) (Ms 4.79; DT $\leq .69)$.

De este modo, encontramos una tendencia general en relación a las percepciones de los participantes, con una calificación positiva de la dimensión personal y de motivación en la tutorización de sus trabajos mientras que se constata la valoración opuesta en la dimensión burocrática, administrativa y emocional del tutor/a, percibiendo que hay gran porcentaje de estudiantes que confirman la escasa adecuación de sus tutores/as para suplir sus necesidades en esta dimensión. Con la intencionalidad de conocer la asociación entre las variables estudiadas para, de este modo, profundizar en las características de la tutorización de TFG y TFM ejercida a lo largo del periodo analizado y mejorar la acción tutorial en este contexto, se procedió la realización del coeficiente de correlación de Spearman (Hernández, Fernández y Baptista, 2010).

Tabla 2. Valores de media (M) y desviación (DT) del instrumento.

\begin{tabular}{|c|c|c|}
\hline Ítems del instrumento analizados & M & DT \\
\hline Ítem 5. La comunicación con mi tutor/a de TFG/TFM ha sido adecuada. & 3.14 & 0.46 \\
\hline Ítem 6. El tutor/a responde a mis consultas online en un tiempo aceptable. & 3.21 & 0.65 \\
\hline $\begin{array}{l}\text { Ítem 7. Las respuestas que he realizado a mi tutor/a a la consultas para el desarrollo de mi TFG/TFM han } \\
\text { sido satisfactorias. }\end{array}$ & 4.59 & 0.44 \\
\hline $\begin{array}{l}\text { Ítem 8. La resolución, por parte de mi tutor/a, de dudas sobre aspectos formales y burocráticos de mi } \\
\text { TFG/TFM, ha sido correcta. }\end{array}$ & 2.01 & 0.61 \\
\hline Ítem 9. Estoy contento/a con el tutor/a asignado/a para mi TFG/TFM. & 3.17 & 0.69 \\
\hline $\begin{array}{l}\text { Ítem 10. Considero que mi tutor/a de TFG/TFM me ha orientado adecuadamente y desde un principio } \\
\text { sobre el trabajo a desarrollar. }\end{array}$ & 4.21 & 0.67 \\
\hline Ítem 11. El tutor/a ha planteado un cronograma de trabajo para mi orientación y desarrollo de mi TFG/TFM. & 4.19 & 0.56 \\
\hline $\begin{array}{l}\text { Ítem 12. Considero que mi tutor/a de TFG/TFM ha empatizado conmigo a lo largo de la tutorización } \\
\text { realizada. }\end{array}$ & 3.19 & 0.74 \\
\hline $\begin{array}{l}\text { Ítem } 13 \text {. Mi tutor/a de TFG/TFM ha conectado emocionalmente conmigo y así lo ha demostrado a lo largo } \\
\text { de la tutorización realizada. }\end{array}$ & 2.88 & 0.81 \\
\hline $\begin{array}{l}\text { Ítem 14. Mi tutor/a de TFG/TFM me ha transmitido confianza y seguridad a lo largo de la tutorización } \\
\text { ejercida. }\end{array}$ & 4.02 & 0.62 \\
\hline $\begin{array}{l}\text { Ítem 15. Considero que mi tutor/a de TFG/TFM me ha aportado estabilidad para poder llevar a } \\
\text { cualquier pregunta que me surgiera, a lo largo de la tutorización ejercida. }\end{array}$ & 4.06 & 0.68 \\
\hline $\begin{array}{l}\text { Ítem 16. Mi tutor/a de TFG/TFM contaba con los conocimientos académicos necesarios para orientarme } \\
\text { en mi investigación. }\end{array}$ & 4.89 & 0.44 \\
\hline $\begin{array}{l}\text { Ítem 17. Mi tutor/a de TFG/TFM contaba con los conocimientos administrativos y burocráticos para } \\
\text { orientarme en mi trabajo. }\end{array}$ & 2.12 & 0.59 \\
\hline $\begin{array}{l}\text { Ítem 18. Mi tutor/a de TFG/TFM contaba con la formación tutorial adecuada para gestionar mis dudas } \\
\text { correctamente. }\end{array}$ & 2.34 & 0.65 \\
\hline $\begin{array}{l}\text { Ítem 19. Mi tutor/a de TFG/TFM me ha motivado y animado para poder llevar a cabo y finalizar mi } \\
\text { investigación. }\end{array}$ & 4.45 & 0.63 \\
\hline $\begin{array}{l}\text { Ítem 20. Mi tutor/a de TFG/TFM ha sido realista y ha reconocido sus propias debilidades a la hora de } \\
\text { orientarme en mi investigación. }\end{array}$ & 4.01 & 0.63 \\
\hline $\begin{array}{l}\text { Ítem 21. Considero que la comunicación con mi tutor/a de TFG/TFM se adecua a mis necesidades para } \\
\text { llevar a cabo mi TFG/TFM. }\end{array}$ & 3.24 & 0.69 \\
\hline $\begin{array}{l}\text { Ítem 22. Mi tutor/a de TFG/TFM ha llevado a cabo un seguimiento de mi investigación durante todo el } \\
\text { proceso de tutorización. }\end{array}$ & 4.45 & 0.48 \\
\hline $\begin{array}{l}\text { Ítem 23. Considero que mi tutor/a de TFG/TFM ha estado pendiente de mi rendimiento a lo largo del } \\
\text { proceso de tutorización. }\end{array}$ & 4.01 & 0.57 \\
\hline $\begin{array}{l}\text { Ítem 24. Mi tutor/a de TFG/TFM me ha ayudado para transformar mis debilidades en fortalezas para poder } \\
\text { llevar a cabo, con éxito, mi investigación. }\end{array}$ & 4.52 & 0.48 \\
\hline $\begin{array}{l}\text { Ítem 25. La retroalimentación recibida de mi tutor/a en cuanto al tiempo de corrección de mi TFG/TFM ha } \\
\text { sido la adecuada. }\end{array}$ & 3.11 & 0.51 \\
\hline Ítem 26. Mi tutor/a de TFG/TFM ha mostrado interés y ha estado atento en todo momento a mi progreso. & 4.64 & 0.55 \\
\hline Ítem 27. Me siento satisfecho/a con el trabajo de tutorización de mi tutor/a de TFG/TFM. & 4.88 & 0.67 \\
\hline Ítem 28. Mi tutor/a de TFG/TFM me ha orientado, incluso, en relación a mi futura labor como & 4.79 & 0.69 \\
\hline
\end{tabular}


En primer lugar, se constata una elevada correlación positiva entre la dimensión organizativa de la acción tutorial (seguimiento de los tutorandos/as, de la orientación y progreso desarrollado en la confección de sus trabajos) con la dimensión académica del tutor/a (formación en los conocimientos curriculares de las investigaciones en desarrollo), con resultados del valor $p \geq 0.771$, cercanos al registro +1 (Hernández, Fernández y Baptista, 2010) e indicativos de la fuerte asociación entre las dimensiones. Similares resultados se obtienen entre la dimensión relacionada con la motivación ejercida por el tutor/a y el seguimiento del trabajo con un resultado de valor $p \geq 0.709$.

En segundo lugar, se confirman una serie de correlaciones de valor medio positivo, de valor $p \leq 0.4$. Es el caso de la correlación entre la dimensión personal (empatía, conexión emocional) y la dimensión comunicativa (resolución de dudas en tiempo y satisfacción en las respuestas obtenidas), que arroja un valor $p \geq 0.328$. En este grupo se halla también la correlación entre la dimensión comunicativa y la dimensión académica con un valor $p \geq 0.357$, ilustrativo de necesidad de promover una mejora en la interacción de la acción tutorial. En tercer lugar, encontramos un bloque de correlaciones negativas, que confirman la asociación inversa entre las dimensiones, es decir que, al aumentar un rango, el otro decrece. Así, se observa este tipo de correlación negativa entre la dimensión en formación en aspectos burocráticos (resolución de dudas sobre formularios y procesos a seguir con la administración educativa) y la valoración general del tutor/a (valor $p \geq-0.211$ ), indicativo de la precariedad en la formación de los tutores en cuanto a temas de funcionamiento y gestión de los centros universitarios. Esto mismo sucede entre la dimensión académica y la gestión de dudas no formativas o de contenidos teóricos (valor $p \geq-0.183$ ), que pone de relieve la carencia en cuanto a la formación del tutor/a para la resolución de dudas no eruditas o doctas.

Respecto al segundo instrumento de estudio, la entrevista individual, se han analizado, cualitativamente, las respuestas en torno a las cinco dimensiones de análisis centrales: dimensión personal (motivación, y promoción positiva de autoconcepto; desarrollo de la autoestima; capacidad y habilidades sociales; integración de los estudiantes en los procesos de TFG y TFM; promoción de la participación en contextos comunitarios del alumnado; resolución de problemas personales o de ajuste académico y social en el plano intelectual, social, emocional, institucional; etc.); dimensión académica (información sobre la asignatura TFG o TFM; resolución de dudas sobre conceptos clave de la investigación en proceso; revisión de la evaluación; conocimiento en teorías y análisis de datos; resolución de dudas sobre contenidos académicos; toma de decisiones frente a epígrafes y estructura del TFG o TFM; etc.); dimensión burocrático-administrativa (información sobre los diferentes servicios de orientación académica, profesional y personal de la universidad y de sus centros; información sobre diferentes tareas administrativas y burocráticas; plazos de defensa y epígrafes de los documentos base a rellenar, etc.); dimensión organizativa y profesionalizante (planificación y organización del TFG o TFM; metodología docente para profundizar en los contenidos abordados en la investigación; diseño de cronogramas y planificación del TFG o TFM; promoción en la transición al mundo laboral; presentación y ayuda en la elección de un proyecto profesional; información sobre el mercado de trabajo y salidas profesionales; promoción del aprendizaje a lo largo de la vida, etc.); y dimensión técnicacomunicativa (seguimiento de los trabajos y los procesos prácticos desarrollados; mejora del aprendizaje; uso adecuada de las tecnologías para la comunicación alumnado-tutor/a; asesoramiento en relación a recursos en la Web e Internet para el proceso investigativo; etc.).

Los datos cualitativos obtenidos por MAXQDA (v. 2018.2) arrojan resultados que convergen con los obtenidos en el análisis cuantitativo del cuestionario o instrumento 1. Con una amplia frecuencia, los estudiantes emplearon los términos empatía, gestión, agilidad en las respuestas, orientación y motivación para referirse a las características principales prioritarias en la acción tutorial de calidad, en el desarrollo de sus TFG o TFM (Tabla 3). La acción de tutorización en TFG o TFM de calidad se asocia a conceptos relacionados con las cinco dimensiones confeccionadas. Si atendemos a los datos de manera pormenorizada, en relación a la dimensión personal, que recoge entre las respuestas dadas las características de: empatía, interactuación con el alumnado, amabilidad del tutor/a con el estudiante, promoción de la motivación del tutorando y apoyo en la elaboración de su investigación, se constata un valor de los descriptivos de alta aproximación a la variable objeto de estudio $\left(M_{0}\right.$ = 3; $D T=0.39)$. De manera específica, destacan las 187 citas $(f=187)$, un $91 \%$ de los estudiantes considera, en el tercer nivel de aproximación, que la empatía es una característica prioritaria de la función tutorial en TFG/TFM (Tabla 3).

La motivación del alumnado es otro de los factores más reconocidos. Este reconocimiento se vincula, mayoritariamente en un nivel alto de aproximación ( $f=159 ; 88 \%)$, con la necesidad de un equilibrio entre la empatía y la motivación para completar la atención del tutorando/a. En esta misma línea, en la dimensión académica, se comprueba la relevancia otorgada a la resolución de dudas $(f=134)$, los conocimientos $(f=108)$ y a la formación $(f=102)$ del tutor/a de TFG/TFM. Estos términos suponen el $85 \%, 80 \%$ y $79 \%$ respectivamente de estudiantes que perciben tales características como relevantes para la acción tutorial $\left(M_{0}=3 ; D T=0.59\right)$. También, en referencia al nivel más elevado de aproximación (valor=3), se encuentra la dimensión técnicocomunicativa. Los análisis realizados permiten constatar la importancia dada a los términos concretos de 
manejo de tecnologías por parte del tutor/a $(f=91)$, donde un $75 \%$ de participantes se refiere a ello como elemento imprescindible en la acción tutorial de TFG/TFM. Asimismo, respuestas ágiles ( $f=89$ ) es citado por el $73 \%$ de los estudiantes como necesario en la caracterización de los tutores/as.

Tabla 3: Frecuencia descriptiva (f) $M_{0}$ : Moda; DT: Desviación típica de dimensiones y términos para la definición de la tutorización de calidad en TFG/TFM aproximación

\begin{tabular}{|c|c|c|c|c|c|}
\hline Dimensión & Término & $f$ & $\%$ & $\mathrm{M}_{0}$ & DT \\
\hline \multirow{5}{*}{ Personal } & Empatía & 187 & $91 \%$ & \multirow{5}{*}{3} & \multirow{5}{*}{0.39} \\
\hline & Interactúa & 121 & $84 \%$ & & \\
\hline & Amable & 93 & $76 \%$ & & \\
\hline & Motivador/a & 159 & $88 \%$ & & \\
\hline & Apoyo & 111 & $81 \%$ & & \\
\hline \multirow{5}{*}{ Académica } & Formación & 102 & $79 \%$ & \multirow{5}{*}{3} & \multirow{5}{*}{0.59} \\
\hline & Conocimientos & 108 & $80 \%$ & & \\
\hline & Recursos & 57 & $51 \%$ & & \\
\hline & Preparación & 57 & $51 \%$ & & \\
\hline & Responder dudas & 134 & $85 \%$ & & \\
\hline \multirow{5}{*}{$\begin{array}{l}\text { Burocrático- } \\
\text { administrativa }\end{array}$} & Información actualizada & 86 & $72 \%$ & \multirow{5}{*}{2} & \multirow{5}{*}{0.72} \\
\hline & Conocer normativa & 34 & $31 \%$ & & \\
\hline & Recursos legales & 45 & $43 \%$ & & \\
\hline & Plazos administrativos & 36 & $34 \%$ & & \\
\hline & Procesos burocráticos & 81 & $69 \%$ & & \\
\hline \multirow{5}{*}{$\begin{array}{l}\text { Organizativa- } \\
\text { profesionalizante }\end{array}$} & Organiza cronograma de entregas & 84 & $71 \%$ & \multirow{5}{*}{2} & \multirow{5}{*}{0.77} \\
\hline & Distribuye los tiempos & 62 & $57 \%$ & & \\
\hline & Orienta sobre los plazos & 37 & $35 \%$ & & \\
\hline & Orienta para la labor docente & 28 & $25 \%$ & & \\
\hline & Informa sobre la labor docente & 46 & $44 \%$ & & \\
\hline \multirow{5}{*}{ Técnica-comunicativa } & Respuestas ágiles & 89 & $73 \%$ & \multirow{5}{*}{3} & \multirow{5}{*}{0.68} \\
\hline & Resolución de dudas & 82 & $70 \%$ & & \\
\hline & Gestión del correo & 78 & $66 \%$ & & \\
\hline & Gestión de la plataforma universitaria & 33 & $31 \%$ & & \\
\hline & Manejo de tecnologías & 91 & $75 \%$ & & \\
\hline
\end{tabular}

A estas percepciones, en el nivel máximo de aproximación, le siguen los términos: información actualizada $(f=86)$ con un $72 \%$ de participantes que consideran una necesaria formación en la dimensión burocráticoadministrativa de los tutores/as, para incrementar la información de los estudiantes y alcanzar una verdadera implicación en su acción tutorial; y organiza cronograma de entregas, con una $f=84$ y un $71 \%$ del alumnado que considera esta características necesaria en los tutores/as para lograr un correcto desarrollo en el proceso de construcción de los TFG/TFM.

A estas percepciones, en el nivel máximo de aproximación, le siguen las consideraciones sobre otros rasgos de la función tutorial, que incrementan la motivación de los estudiantes y permiten la consecución de los objetivos didáctico-formativos implicados en la elaboración de TFG o TFM. Tal es el caso de los conceptos como: interactuación $(f=121 ; 84 \%)$, apoyo $(f=111 ; 81 \%)$ y amabilidad $(f=93 ; 76 \%)$ con el estudiante en la dimensión personal analizada; resolución de dudas $(f=82 ; 70 \%)$ y la gestión del correo $(f=78 ; 66 \%)$ en la dimensión técnico-comunicativa. En el segundo nivel de aproximación (valor $\mathrm{M}_{0}=2$ ) se encuentra, de manera general, la dimensión organizativa-profesionalizante y la burocrática administrativa. Para la primera de ellas, los términos orienta para la labor docente $(f=28 ; 25 \%)$ y orienta sobre los plazos $(f=37 ; 35 \%)$ presentan una frecuencia de aparición muy baja así como una representatividad también muy débil en las respuestas de los estudiantes. Situación similar se observa en la dimensión burocrático-administrativa, con frecuencias muy bajas en conocer normativa $(\mathrm{f}=34 ; 31 \%)$ y plazos administrativos $(f=36 ; 34 \%)$ de escasa representatividad en el grueso de las contestaciones emitidas.

En la figura 1, resultado de la opción Árbol de palabras del programa cualitativo MAXQDA, se pueden visualizar los distintos términos así como su mayor o menor prevalencia y las uniones de tales conceptos, entre sí, tras las entrevistas realizadas.La figura ha sido mejorada, se ha utilizado letra Arial tamaño 8 pto. Como señala el revisor en las letras de manera que sí que se puedan leer. El problema de visualización es que, al tratarse de una imagen y ocupar el espacio de formato papel vertical, no se puede ampliar más. 


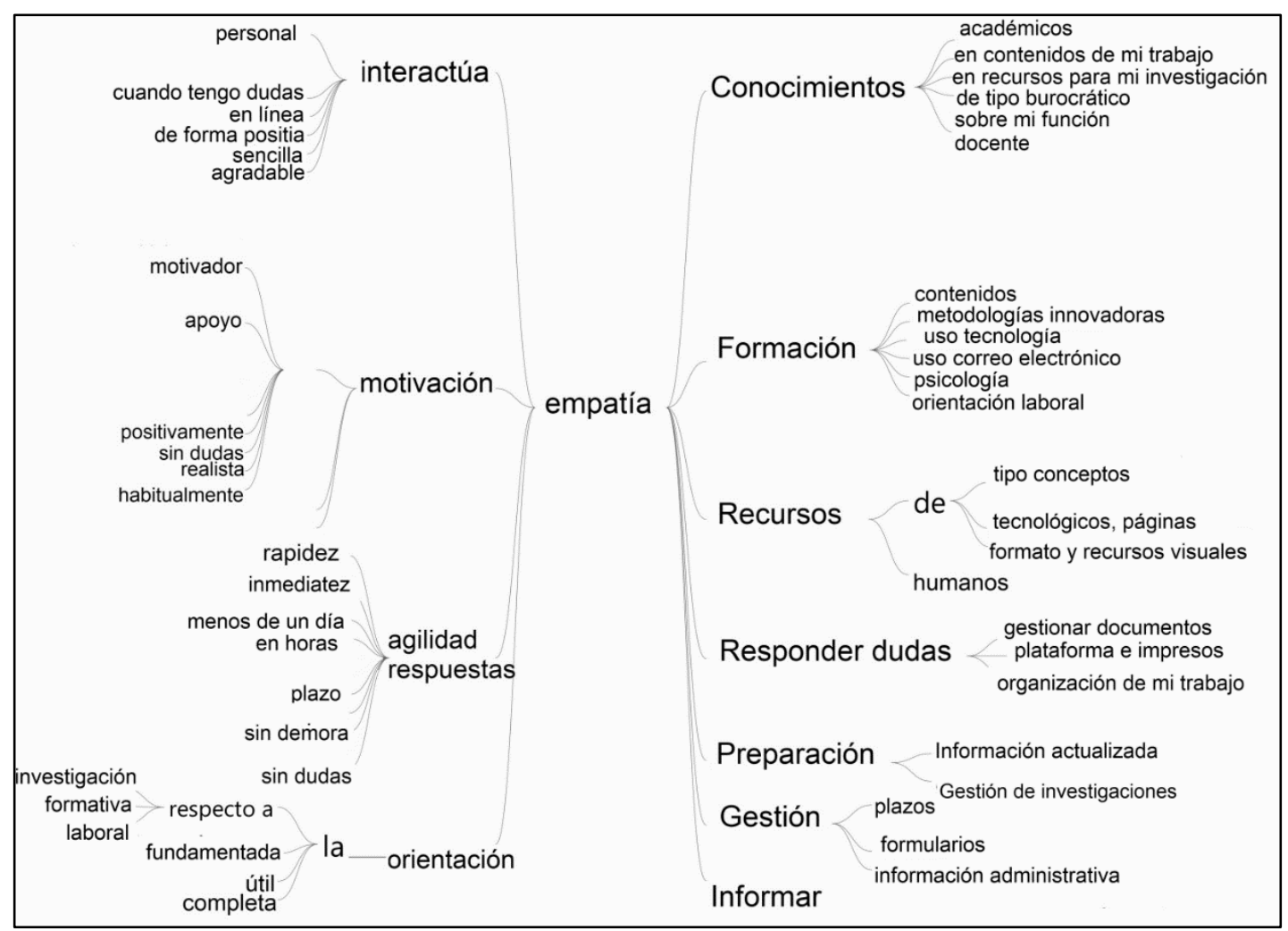

Fig. 1: Árbol de palabras relativo a la frecuencia $(f)$ de aparición según MAXQDA 2018.2.

Destaca, grosso modo, la importancia central del término empatía, de la dimensión personal y la íntima relación detectada con los conceptos interactúa, motivación, agilidad de respuestas, orientación, conocimientos, formación, recursos, responder dudas, preparación, gestión e informar. Como se observa en la figura 1, todos estos términos engloban un tercer nivel, relacionado con aspectos más concretos, que el alumnado participante reconoce como clave para la calidad de la tutorización en TFG/TFM.

\section{DISCUSIÓN}

Los nuevos requerimientos del rol docente así como la compleja tarea de tutorizar al alumnado universitario convergen en una dificultad añadida cuando esta acción tutorial, de acompañamiento y orientación, se circunscribe al contexto de la elaboración de TFG/TFM. El tutor/a se perfila como el profesional que ha de encargarse de formar en situaciones significativas de investigación y, a la vez, debe ocuparse de los aspectos de orientación personal, académica y profesional de sus estudiantes (Alonso-García et al., 2018). Tales imperativos de transformación se han visto reflejados en las propuestas europeas, en las que la función tutorial adquiere un lugar importante en la labor del docente universitario, y en las que se trata de dar respuesta a aspectos (o dimensiones) múltiples. En este sentido, se señalan algunas de estas nuevas tareas del tutor/a universitario como son: los servicios de orientación, las tareas administrativas, la formación en conflictos entre estudiantes y profesorado, las habilidades sociales que generen un ambiente positivo y cooperativo entre iguales, la cualificación en aspectos motivacionales, de gestión de autoestima, la formación en el desarrollo profesional del estudian, entre otras (Hernández et al., 2016; Winder et al., 2016; Alonso-García et al., 2018; Juan et al., 2018).

A partir de los resultados cuantitativos obtenidos y las respuestas expuestas por los participantes en la investigación, así como la revisión de aquellos autores que han tratado esta misma temática, se observa una línea común en relación con aquellos factores y elementos que señalan una tutorización de calidad. Destaca la importancia relativa al conocimiento especializado en las áreas de intervención (académica, profesional), así como por aspectos personales que permiten su desarrollo, tales como empatía, compromiso y motivación, unidos a la competencia en conocimientos técnicos y comunicativos para el que deben estar formados los tutores/as de TFG/TFM. Tales aspectos son destacados por los participantes en este trabajo. Estos resultados coinciden con las investigaciones de Coll et al. (2014) donde se señala que la acción de tutorización, como es el caso, no se basa únicamente en el mejoramiento de las técnicas de investigación, búsqueda de información y elaboración de resultados, sino en la acción orientadora, responsabilidad fundamental de todo docente-tutor/a de TFG/TFM. Del mismo modo, se pone de relieve la necesidad de resolver cuestiones relativas a aspectos personales y emocionales de los estudiantes durante el desarrollo de la elaboración de 
sus TFG/TFM como principal objetivo de la tarea del docente-tutor/a. Esta dimensión personal ha sido destacada ya por otros autores como Juan et al. (2018) y quienes confirman que gran parte del alumnado valora positivamente la utilidad del espacio de tutoría para el desarrollo personal y para la resolución de dificultades emocionales surgidos al amparo del propio proceso de enseñanza y aprendizaje.

Por otro lado, uno de los aspectos en donde los tutorandos/as llaman la atención es en el tiempo de respuesta o feedbak tutor/a-estudiante. Los participantes indican la comunicación como una de las principales cualidades de una tutorización de calidad. En esta línea, Aguilar-Salinas, Chávez-Valenzuela, y de las Fuentes-Lara (2017), ya señalan como primordial ajustar el número de estudiantes por docente-tutor/a, de manera que se lleve a cabo una correcta acción tutorial. También, Ferguson (2011) y García et al. (2012), inciden en los aspectos temporales, de respuestas, constatando la valoración positiva del alumnado cuando se implementan programas de tutorías concretos, con cronogramas claros. De una manera más pormenorizada, los valores obtenidos apuntan hacia la necesidad de que los tutores/as dispongan de una adecuada formación en el manejo de las TIC (Ortega-Sánchez y Gómez-Trigueros, 2019; Gómez-Trigueros et al., 2019). Destaca la relevancia otorgada al manejo de las tecnologías para el desarrollo de la acción tutorial de calidad. Es pues preciso hacer hincapié en el desarrollo de diferentes medios de atención al estudiante, siendo la tutoría online uno de los sistemas más idóneos para desarrollar un mayor compromiso del estudiante con su aprendizaje (ChanLin, 2016; Alonso-García et al., 2018).

Otro aspecto que se pone de relieve en este trabajo es la dimensión académica del tutor/a. Los resultados arrojan una valoración muy positiva en relación a la importancia de la formación y los conocimientos académicos del profesorado para poder, de este modo, resolver dudas que puedan surgir a lo largo del TFG/TFM. Alonso-García et al. (2018) resaltan que los estudiantes ponen en valor la cualificación científica del tutor/a ante la necesidad de obtener respuestas claras sobre la construcción del marco teórico de sus investigaciones en curso (TFG/TFM). Este aspecto también destaca en el trabajo de Coll et al., (2014), que insiste en la importancia de la formación y adecuación del tutor/a a la temática investigativa del estudiante. También, el trabajo de Juan et al. (2018) incide en la importancia de la función tutorial para dar amparo a problemas de carácter instruccional, relacionado con aspectos metodológicos, que puedan aparecer a lo largo del TFG/TFM.

Otro factor estudiado, de un menor peso en la percepción de los participantes, es la dimensión burocráticoadministrativa del tutor/a. Si bien es cierto que se considera meritorio la formación en cuestiones de plazos y entregas de los TFG/TFM por parte de la administración universitaria, este aspecto queda relegado a un segundo plano, eclipsado por otras características ya descritas. Estos resultados coinciden con los de otros autores (Aguilar-Salinas et al., 2017; Amor-Almedina y Dios-Sánchez, 2017; Alonso-García et al., 2018) quienes manifiestan que, a pesar de los cambios introducidos por el EEES y el Plan Bolonia, los estudiantes siguen asociando la tutoría universitaria como una actividad orientada a la resolución de dudas académicas o personales.

De manera similar, los participantes otorgan una menor importancia a la orientación profesional que pueda llevar a cabo el tutor/a de TFG/TFM. Esta percepción es coherente con el objetivo del TFG/TFM, que no se dirige a la formación de profesionales docentes sino a la finalización de unos estudios (Grado y Máster respectivamente). Por el contrario, la acción organizativa y el diseño de cronogramas para el desarrollo de trabajo-investigación sí que arroja resultados elevados, muy en coherencia con el resto de respuestas realizadas coincidiendo con las propuestas y diseños sobre la acción tutorial de otros autores para el caso de las tesis (Hernández et al., 2016) y de los TFG (Juan et al., 2018).

Como valoración general de resultados obtenidos, los participantes muestran satisfacción con el docentetutor/a de su TFG/TFM. Aunque se han obtenido resultados positivos en las dimensiones del estudio, se percibe un gran número de estudiantes que manifiestan la necesidad de incorporar nuevas cualificaciones para una acción tutorial de calidad, que satisfaga las necesidades del alumnado de TFG/TFM, especialmente en el ámbito personal, burocrático-administrativo y comunicativo. De igual forma, se sugiere que se trabaje la formación del tutor/a universitario con la intencionalidad de mejorar e impulsar el desarrollo de sus trabajos de investigación y su posterior defensa; satisfacer las particularidad que emane de las los ajustes y modificaciones de los currículos al EEES (Ceballos, 2017) así como proporcionar habilidades, actitudes y aptitudes para su desarrollo personal como profesional (López-Gómez, 2017; Alonso-García et al., 2018).

\section{CONCLUSIONES}

A partir de los resultados obtenidos, se pueden extraer las siguientes conclusiones:

1) La investigación muestra que, en general, los estudiantes participantes están contentos con la función tutorial recibida a lo largo de la elaboración de su TFG o TFM. Sin embargo, es necesario incidir en ciertos aspectos o características de los tutores/as para poder recibir una tutorización de calidad. 
2) A partir de las respuestas recibidas y de las entrevistas realizadas en esta investigación, se diseña una imagen clara de aquellas características definitorias de un docente-tutor de calidad. Entre las cualidades consideradas por los participantes destacamos: empatía, motivación, resolución de dudas, formación académica, agilidad de respuestas a las dudas, amabilidad, apoyo para poder ofrecer una buena orientación de sus TFG y TFM.

3) La acción tutorial en TFG y TFM debe concebirse como un conjunto de procesos encaminados a la orientación no sólo académica y metodológica, sino también y muy especialmente, personal del alumnado. En este sentido, es necesario diseñar planes formativos, de tutores/as de TFG/TFM, que contemplen la adquisición de conocimientos psicológicos, de gestión de emociones y de orientación personal.

4) Es necesario dotar al profesorado tutor/a de TFG/TFM de una cualificación en competencias digitales, que posibilite una comunicación virtual, fluida y completa. Se sugiere la implementación de talleres relacionados con la formación en competencias digitales docentes, que permita el uso de diversos software y hardware para agilizar los feedback tutor/a-tutorando/a.

5) Se debe considerar la tutorización de TFG/TFM como una acción más compleja, que necesita de una elevada dosis de formación personal y profesional del docente, que sea capaz de acompañar al estudiante en su proceso de investigación, de búsqueda de información, de organización de su trabajo; dotándolo de una autoafirmación, independencia y reflexión para facilitar su progreso personal y académico de final de estudios (de Grado o de Máster según el caso).

6) A través de la bibliografía estudiada, y aunque el trabajo se circunscribe a España, investigaciones realizadas en América Latina arrojan percepciones similares e insisten en la necesidad de la adecuada formación del profesor/a-tutor/a.

\section{REFERENCIAS}

Aguilar-Salinas, W.E., Chávez-Valenzuela, G. E., y de las Fuentes-Lara, M., Tutorías: Estudio Exploratorio sobre la Opinión de los Estudiantes de Tronco Común de Ciencias de la Ingeniería, doi: 10.4067/S0718-50062017000300008, Formación Universitaria, 10(3), 69-80 (2017)

Amor-Almedina, M. I., y Dios-Sánchez, I., La tutoría universitaria: un espacio para la orientación personal, académica y profesional en la formación inicial del profesorado, doi: 1.2017.19362, Rev. Española de Orientación y Psicopedagogía, 28(1), 119-130 (2017)

Alonso-García, S., Rodríguez-García, A.M., y Cáceres-Reche, M.P., Análisis de la Acción Tutorial y su Incidencia en el Desarrollo Integral del Alumnado. El Caso de la Universidad de Castilla La Mancha, España, doi: 10.4067/S071850062018000300063, Formación Universitaria, 11(3), 63-72 (2018)

Ceballos, N., La tutoría como espacio de democracia: una mirada desde la voz de los docentes en formación, doi: 10.5944/reop.vol.28.num.1.2017.19360, Revista Española de Orientación y Psicopedagogía, 28(1), 86-103 (2017)

Coll, C., Rochera, M.J., y De Gispert, I., Supporting online collaborative learning in small groups: Teacher feedback on learning content, academic task and social participation, doi: 10.1016/j.compedu.2014.01.015, Computers \& Education, 75, 53-64 (2014)

ChanLin, L. J., Students' Involvement and Communit Support for Service Engagement in Online Tutoring, doi: 10.6120/JoEMLS.2016.532/0006.RS.CE, Journal of Educational Media y Library Sciences, 53(2), 245-268 (2016)

Chianese, G., The tutorship function: Clifelong learning and transition paths, doi: 10.20319/pijss.2017.s31.775786, International Journal of Social Sciences, 3(1), 775-786 (2017)

De la Cruz, G., García, T., y Abreu, L., Modelo integrador de la tutoría: de la dirección de tesis a la sociedad del conocimiento, ISSN: 1405-6666, Revista Mexicana de Investigación Educativa, 11(31), 1363-1388 (2006)

De Pablos, J., Colás-Bravo, P., Ramírez, T.G., y Cortés, R.J., La adaptación de las universidades al Espacio Europeo de Educación Superior. Un proceso metodológico para el diseño de planes estratégicos, ISSN: 0212-4068, Revista de Investigación Educativa, 25(2), 533-554 (2007)

Díaz, C., y Bastías, C., Una aproximación a los patrones de comunicación entre el profesor mentor y el profesor-estudiante en el contexto de la práctica pedagógica, doi: 10.5944/educxx1.15.1.158, Revista Educación XX1, 15(1), 241-263 (2012)

Dopico, E., Tutoría universitaria: propuestas didácticas de competencia tutorial, doi: 10.4995/redu.2013.5573, Revista de Docencia Universitaria, 11(2), 195-220 (2013)

Dunworth, K., y Santiago, H., Perceptions of quality in staffstudent written feedback in higher education: a case study, doi: 10.1080/13562517.2016 .1160219, Teaching in Higher Education, 21(5), 576-589 (2016)

Ferguson, P., Student perceptions of quality feedback in teacher education, doi: 10.1080/02602930903197883, Assessment \& Evaluation in Higher Education, 36(1), 51-62 (2011) 
García, R., Cuevas, O., Vales, J., y Cruz, I., Impacto de la tutoría presencial y virtual en el desempeño académico de alumnos universitarios, doi: 10.35362/rie5821447, Revista Iberoamericana de Educación, 58(2), 1-11 (2012)

Gómez-Trigueros I.M., Ruiz-Bañuls M., y Ortega-Sánchez D., Digital Literacy of Teachers in Training: Moving from ICTs (Information and Communication Technologies) to LKTs (Learning and Knowledge Technologies), doi:10.3390/educsci90402742019, Education Sciences, 9(4):274 (2019)

Hernández, R., Fernández, C., y Baptista, Maㅡ del P., Metodología de la investigación, McGraw-Hill Educación, México, D.F. (2010)

Hernández, C.A., Jiménez, M., Guadarrama, E., y Rivera, A.E., La Percepción de la Motivación y Satisfacción de la Tutoría Recibida en Estudios de Posgrado, doi: 10.4067/S0718-50062016000200006, Formación Universitaria, 9(2), 49-58 (2016)

Hounsell, D., McCune, V., Hounsell, J., y Litjens, J., The quality of guidance and feedback to students, doi: 10.1080/07294360701658765, Higher Education Research \& Development, 27(1), 55-67 (2008)

Juan, N., Rochera, M.J., Remesal, A. y de Salvadro, N., Qué dificultades perciben los futuros maestros y sus profesores acerca del feedbak recibido durante el Trabajo Final de Grado, doi: 10.4151/07189729-vol.57-iss.1-art.617, Perspectiva Educacional. Formación de Profesores, 57(1), 24-49 (2018)

Lee, H.-J., Hong, T., y Choi, H., Perceptions of tutoring roles and pschological distance among instructors, tutors and students at a Korean universit, doi: 10.1080/07294360.2016.1177811, Higher Education Research y Development, 36(1), 143-157 (2017).

López-Gómez, E., El concepto y las finalidades de la tutoría universitaria: una consulta a expertos, ISSN: 1139-7853, Revista Española de Orientación Psicopedagogía, 28(2), 61-78 (2017)

Mouraz, A., y Sousa, A., An Institutional Approach to First-ear Adjustment, doi: 10.1177/1538192715592928, Journal of Hispanic Higher Education, 15(3), 221-239 (2016)

Ortega-Sánchez, D., y Gómez-Trigueros, I.M., Massive Open Online Courses in the Initial Training of Social Science Teachers: Experiences, Methodological Conceptions, and Technological Use for Sustainable Development, doi: 10.3390/su11030578, Sustainability, 11(3), 578 (2019)

Phillips, F., y Wolcott, S., Effects of Interspersed versus Summary Feedback on the Quality of Students' Case Report Revisions, doi: 10.1080/09639284.2013.847328, Accounting Education, 23(2), 174-190 (2014)

Tralongo, S., Fabriquer / devenir un enseignant du supérieur professionnalisant. Le tutorat en IUT, ISSN: 2107-0946, Formation emploi, 138(2), 165-183 (2017)

Wilcox, R., Modern statistics for the social and behavioral sciences: A practical introduction, Boca Raton, FL: CRC Press, (2011)

Winder, R., Kathpalia, S. S., y Koo, S. L., Writing centre tutoring sessions: addressing students' concerns, doi:10.1080/03055698.2016.1193476, Educational studies: 42(4), 323-339 (2016)

Zohrabi, M., Mixed Method Research: Instruments, Validity, Reliability and Reporting Findings, doi: 10.4304/tpls.3.2.254262, Theory and Practice in Language Studies, 3(2), 254-262 (2013) 